\title{
Comparison of T-cell receptor $\beta$ chain variable region 23-1 open reading frame and 3-1 $F$ CDR3 repertoire in cDNA and gDNA from peripheral blood mononuclear cells of healthy volunteers by high-throughput sequencing
}

\author{
XIAOYAN HE ${ }^{I^{*}}$, BIN SHI ${ }^{*}$, LONG MA ${ }^{l^{*}}$, RUI MA ${ }^{l}$, AIHUA PENG ${ }^{l}$, SUHONG SUN ${ }^{3}$, XINSHENG YAO \\ 'Department of Immunology, Research Center for Medicine \& Biology, Innovation \& Practice Base for Graduate Students Education, \\ Zunyi Medical University, Zunyi, China \\ ${ }^{2}$ Department of Laboratory Medicine, Zunyi Medical University, Zunyi, China \\ ${ }^{3}$ Department of Breast Surgery, the First Affiliated Hospital of Zunyi Medical University, Zunyi, China \\ "These authors contributed equally to this work.
}

\begin{abstract}
The TRBV germline gene can be classified into three types: open reading frame $(O R F)$, functional gene $(F)$, and pseudogene $(P)$; however, the differences and connections among ORF, functional gene, and pseudogene rearrangements and expression characteristics remain unclear. Here, we compared the characteristics of the CDR3 repertoire that was rearranged by TRBV23-1(ORF) and TRBV3-1(F) in four cDNA samples and six gDNA samples from healthy volunteers. In this study, we show that the frequencies of in-frame sequences in the TRBV23-1 complementarity determining region 3 (CDR3) repertoire were significantly lower than those in the TRBV3-1 CDR3 repertoire. The TRBV23-1 CDR3 repertoire, which differed from the TRBV3-1 in-frame and out-of-frame CDR3 repertoire, consisted of 1/3 in-frame sequences and 2/3 out-of-frame sequences. The usage of TRBD1 was higher than that of TRBD2 in the TRBV23-1 in-frame and out-of-frame CDR3 repertoire. In four cDNA samples from PBMCs, the TRBV23-1 in-frame and out-of-frame CDR3 repertoire showed a longer N2 relative to that of N1. Therefore, our results demonstrate that the mechanisms of rearrangement in the TRBV23-1 and TRBV3-1 CDR3 repertoire were different, and thus offered new ideas and methods to resolve the discrepancies in reports on the functionality of TRBV23-1 and to better understand the mechanisms underlying VDJ recombination.
\end{abstract}

Key words: T-cell receptor, T-cell receptor $\beta$ variable, complementarity-determining region 3 repertoire, open reading frame, high-throughput sequencing.

(Centr Eur J Immunol 2018; 43 (3): 295-305)

\section{Introduction}

T-cell receptors (TCRs) are either TCR $\alpha \beta$ or TCR $\gamma \delta$ heterodimers that are formed by four types of peptide chains $\alpha, \beta, \gamma$, and $\delta$. Each polypeptide chain in the thymus rearranges from the TCR germline gene to form a diverse CDR3 repertoire. According to the definition and annotation of the IMGT and EMBL, the TCR germline gene entity can be defined according to three types: open reading frame $(O R F)$, functional gene $(\mathrm{F})$ and pseudogene $(\mathrm{P})$. An open reading frame is a portion of a gene's sequence that contains a sequence of bases, uninterrupted by stop sequences, that could potentially encode a protein. A functional gene's coding region has an $O R F$ without a stop codon, and for which there is no described defect in the splicing sites, recombination signals, or regulatory elements. Pseudogenes are the remnants of genomic sequences of genes which are no longer functional; they are frequent in most eukaryotic genomes, and an important resource for comparative genomics [1-4].

In the process of TCR creation, according to the principle of allelic exclusion, the $\beta$ chain is rearranged earlier than the $\alpha$ chain, so detecting a $\beta$ chain could reflect the

Correspondence: Dr. Xinsheng Yao, Department of Immunology, Zunyi Medical University, 563000 Zunyi, China,

e-mail: immunology01@126.com

Submitted: 27.03.2017; Accepted: 31.07.2017 
character of TCR. According to the homology of the human $T R B V$ gene, $65 T R B V$ genes can be divided into 24 conservative families, which can cover more than $85 \%$ of human TCR $\beta$ chain genes. The complementarity-determining region 3 (CDR3) of a specific TCR $\beta$ chain could be amplified through designing sense primers and TRBC antisense primers. According to the definition and annotation of the IMGT, the human TCR $\beta$ locus consists of $48 T R B V$ functional genes, 19 TRBV pseudogenes, and 7 ORFs. However, some $T R B V$ genes are difficult to classify. For example, $T R B V 7-3$ is classified into either a functional gene or an $O R F$, namely, TRBV7-3*01, TRBV7-3*04 and TRBV7$3 * 05$ (accession numbers X61440, X74843 and M13550, respectively), whereas $T R B V 7-3 * 02$ and $T R B V 7-3 * 03$ are classified into ORFs (accession numbers M97943 and AF009660). TRBV16 is classified into either a functional gene or a pseudogene: $T R B V 16^{*} 01$ and $T R B V 16^{*} 03$ are classified into a functional gene (accession numbers L26231 and L26054), whereas TRBV16*02 is classified into a pseudogene (accession number U03115) [3, 4]. Identification of the functions of these TRBVs is highly complex.

There is currently a debate on how to define and classify human TRBV19S1 (TRBV23-1), and the human T-cell receptor $\beta$ chain variable region (clone HVB10.1) was initially defined as a pseudogene, then later described as an $O R F$ because nucleotide bases $G T$ were replaced by $A T$ in the donor splice site, whereas only a portion of the V-gene has been identified [3]. In 1996, Currie et al. [5] discovered a single extra base in the TRBV19S1 leader sequence, which leads to reading frame changes after mRNA splicing, thereby resulting in premature termination of mRNA translation. Therefore, the TRBV19S1 would be non-functional after rearrangement. However, in 1999, while conducting a research study involving children infected with HIV, Than et al. [6] discovered that TRBV19S1 had dominant usage expression, thus prompting him to suggest that these T-cells participate in the immune response. In 1999, Manfras et al. [7] sequenced more than 500 TRBV20S1 and TRBV19S1 genes. Their study identified different CDR3 lengths between the productive sequences (TRBV20S1) and in-frame nonproductive sequences (TRBV19S1), although no significant differences in TRBD and TRBJ usage and nucleotide additions in junctional sequences were observed.

These analyses were based on the intrinsic defects in gene sequences or by cloning a specific CDR3 region to indirectly demonstrate the in-frame and out-of-frame rearrangements of TRBV23-1. The mechanism underlying the participation of $T R B V 23-1$ in the VDJ rearrangement, and the characteristics of CDR3 repertoires rearranged by TRBV23-1 as well as differences in CDR3 repertoires rearranged by a $T R B V$ functional gene have not been elucidated to date. In this study, we compared the characteristics of the CDR3 repertoire that has been rearranged by TRBV23-1 $(O R F)$ with that by $T R B V 3-1(\mathrm{~F})$ in the cDNA samples from four healthy volunteers by laser capillary electrophoresis scanning and 454 GS FLX high-throughput sequencing (HTS). We also compared the characteristics of the CDR3 repertoire that has been rearranged by TRBV23-1 with that by $T R B V 3-1$ in the gDNA samples from six healthy volunteers by Illumina sequencing. Our results show that the frequencies of in-frame sequences in the TRBV23-1 CDR3 repertoire were significantly lower than those in the TRBV3-1 CDR3 repertoire. The TRBV23-1 CDR3 repertoire, which differed from the TRBV3-1 in-frame and out-of-frame CDR3 repertoire, consisted of $1 / 3$ in-frame sequences and 2/3 out-of-frame sequences. The usage of TRBDI was higher than that of TRBD2 in the TRBV23-1 in-frame and out-of-frame CDR3 repertoire. In four cDNA samples from PBMCs, the TRBV23-1 in-frame and out-of-frame CDR3 repertoire showed a longer $\mathrm{N} 2$ relative to that of $\mathrm{N} 1$. The mechanisms of rearrangement in the TRBV23-1 (ORF) and TRBV3-1 (F) CDR3 repertoire were different.

\section{Material and methods}

\section{The CDR3 repertoire of $T R B V 23-1$ and TRBV3-1 in the cDNA samples from PBMCs of four healthy volunteers}

Ten-milliliter peripheral blood samples were collected from each of four healthy volunteers (H-1: male, 14 years old; H-2: female, 40 years old; H-3: male, 13 years old; $\mathrm{H}-4$ : male, 13 years old). $\mathrm{H}-3$ and $\mathrm{H}-4$ were a pair of twins. H-2 was a mother of twins. H-1 was the same age as the twins. Peripheral blood mononuclear cells (PBMCs) were separated by Ficoll-Hypaque density gradient centrifugation. The study protocol was approved by the local ethics committee. The volunteers had signed the informed consent, and in the case of the younger subjects under the age of 15 , they also had the signed informed consent from their parents. Total RNA was extracted from each of the four PBMCs samples, and cDNA was prepared. Using the $T R B C$ downstream primer with the FAM label and the individual $T R B V$ upstream primers, the TRBV CDR3 regions were amplified by PCR ( 1 cycle of $94^{\circ} \mathrm{C}$ for $3 \mathrm{~min}, 1$ cycle; 35 cycles each of $94^{\circ} \mathrm{C}$ for $60 \mathrm{~s}, 55^{\circ} \mathrm{C}$ for $60 \mathrm{~s}$, and $72^{\circ} \mathrm{C}$ for $60 \mathrm{~s} ; 1$ cycle of $72^{\circ} \mathrm{C}$ for $10 \mathrm{~min}$ ). The PCR products were stored at $-20^{\circ} \mathrm{C}$.

Laser capillary electrophoresis DNA scan (ABI3730) was used to analyze each PCR product of the TRBV gene CDR3. Peak Scanner Software v1.0 was used to analyze the CDR3 spectratyping. The TRBV23-1 and TRBV3-1 CDR3 PCR products of 4 healthy volunteers were chosen for cloning and sequence analysis of in-frame or out-of-frame rearrangements that consisted of the CDR3-region (Fig. 1).

Using the four sets of upstream and downstream primers with specific base labels (Table 1), the CDR3 regions of the $T R B V$ genes were separately amplified by polymerase chain reaction (PCR; using the same conditions as earlier described). Roche 454 GS FLX high-throughput 
sequencing was used to determine the genomic sequences of the CDR3 repertoire (the PCR products) of each sample by Majorbio Bio-pharm Technology Co., Ltd. (Shanghai, China). IMGT High V-QUEST was used to screen the original HTS data (Fig. 1). First, "Unproductive", "Warnings", "Unknown functionality", and "No results" sequences were removed. Then, the in-frame and out-offrame CDR3 sequences with a V-gene consistency rate of $>90 \%$ were selected for analysis [8-11].
The CDR3 repertoire of TRBV23-1 and TRBV3-1 in the gDNA samples from PBMCs of six healthy volunteers

Ten-milliliter samples of peripheral blood were collected from each of six healthy volunteers (H-5: male, 46 years old; H-6: male, 46 years old; H-7: male, 35 years old; H-8: male, 35 years old; $\mathrm{H}-9$ : male, 22 years old; $\mathrm{H}-10$ : female, 22 years old). The study protocol was approved by the lo-

Homo sapiens TRB

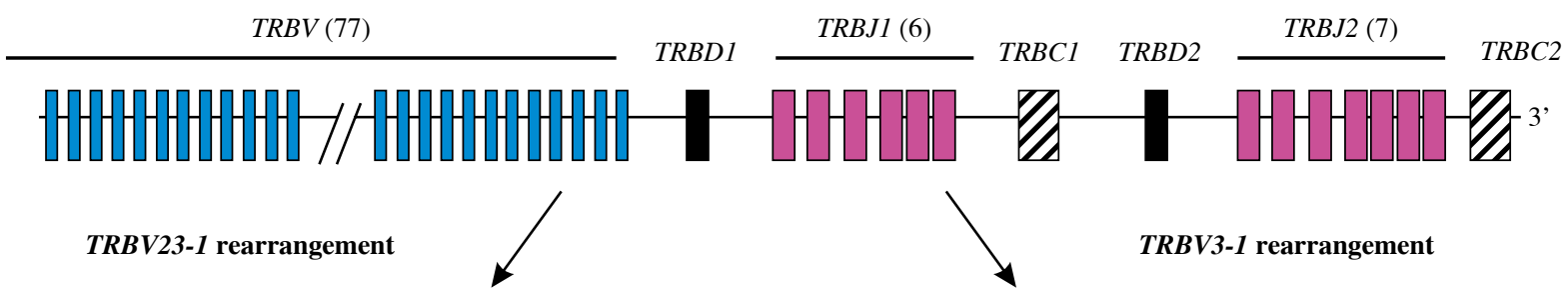

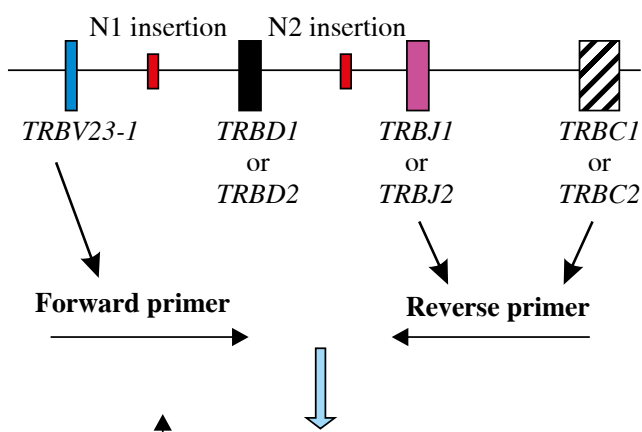

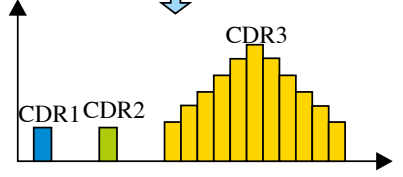

Laser capillary electrophoresis CDR3 spectratyping

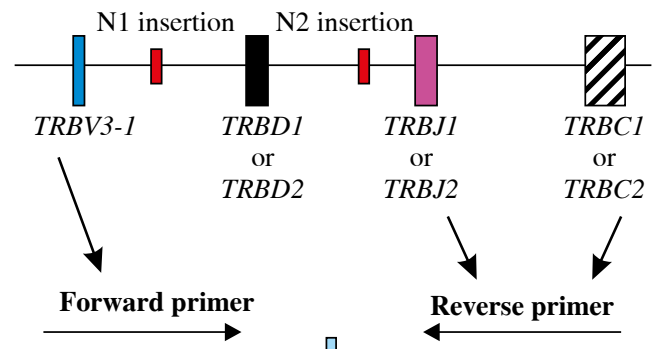

PCR CDR3 products
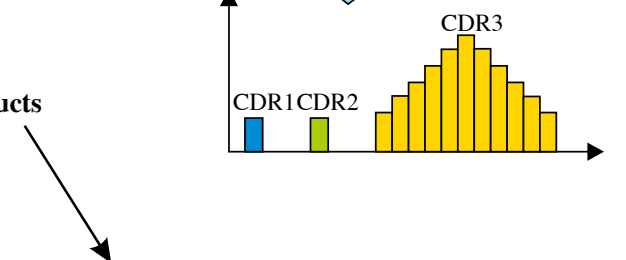

High-throughput sequencing

CDR3 repertoire

Fig. 1. The TRBV CDR3 rearrangements principle and the experimental procedure of the CDR3 repertoire spectratyping analysis by laser capillary electrophoresis and HTS

Table 1. Primer sequences of TCR- $\beta$ chain TRBV3-1 and TRBV23-1 and TRBC in human for 454 GS FLX high-throughput sequencing

\begin{tabular}{|c|c|c|c|}
\hline & TRBV3-1 & $T R B V 23-1$ & $T R B C$ \\
\hline $\mathrm{H}-1$ & $\begin{array}{c}\text { GAGTGCGTCT } \\
\text { ctaaatctccagacaaagctcac }\end{array}$ & $\begin{array}{c}\text { GAGTGCGTCT } \\
\text { caatgccccaagaacgcaccetgc }\end{array}$ & $\begin{array}{l}\text { AGACGCACTC } \\
\text { ttctgatggctcaaacac }\end{array}$ \\
\hline H-2 & $\begin{array}{c}\text { CTACAGTGCT } \\
\text { ctaaatctccagacaaagctcac }\end{array}$ & $\begin{array}{c}\text { CTACAGTGCT } \\
\text { caatgccccaagaacgcaccetgc }\end{array}$ & $\begin{array}{l}\text { AGCACTGTAG } \\
\text { ttctgatggctcaaacac }\end{array}$ \\
\hline $\mathrm{H}-3$ & $\begin{array}{c}\text { CGTGTCTGAT } \\
\text { ctaaatctccagacaaagctcac }\end{array}$ & $\begin{array}{c}\text { CGTGTCTGAT } \\
\text { caatgccccaagaacgcaccetgc }\end{array}$ & $\begin{array}{l}\text { ATCAGACACG } \\
\text { ttctgatggctcaaacac }\end{array}$ \\
\hline $\mathrm{H}-4$ & $\begin{array}{c}\text { CTCGCGATAT } \\
\text { ctaaatctccagacaaagctcac }\end{array}$ & $\begin{array}{c}\text { CTCGCGATAT } \\
\text { caatgccccaagaacgcaccetgc }\end{array}$ & $\begin{array}{l}\text { ATATCGCGAG } \\
\text { ttctgatggctcaaacac }\end{array}$ \\
\hline
\end{tabular}


cal ethics committee. The subjects had signed the informed consent. PBMCs were isolated by Ficoll-Hypaque density gradient centrifugation and genomic DNA was extracted from the six samples, yielding the following individual total amounts: H-5, 1,800 ng; H-6, 1,990 ng; H-7, 3,350 ng; H-8, 5,090 ng; H-9, 2,380 ng; and H-10, 1,730 ng.

The CDR3 repertoire of human TCR beta chain preparation and Illumina HTS sequencing of the gDNA samples were completed by Adaptive Biotechnologies Corp [12]. Adaptive Biotechnologies has developed a novel method that amplifies rearranged TCR CDR3 sequences and exploits the capacity of high-throughput sequencing technology to sequence tens of thousands of TCR CDR3 chains simultaneously (Fig. 1). Because the technology utilizes gDNA, the frequency of sequenced CDR3 chains is representative of the relative frequency of each CDR3 sequence in the starting population of T-cells [13-15]. The CDR3 sequences were screened and analyzed using the ImmuneSEQ assay [16] and the IMGT High V-QUEST [3]. The CDR3 sequences of TRBV3-1 and TRBV23-1 were selected from all the CDR3 repertoire sequences for analysis. First, 'Unproductive', 'Warnings', 'Unknown functionality', and 'No results' sequences were removed. Then, the in-frame and out-of-frame CDR3 sequences with a V-gene consistency rate of $>90 \%$ were selected for analysis $[14,15]$.

\section{Experimental reagents and statistical analysis}

Total RNA and total DNA extraction reagent kits, the gel extraction kit, the PCR purification kit, the Taq PCR master mix, and the DNA extraction and freeze-dry kit were obtained from Qiagen (Germany). DNA marker,
DNA loading buffer, and cDNA synthesis kits were obtained from MBI Fermentas (Canada).

$T R B D$ gene and TRBJ gene usage were compared using the $\chi^{2}$ test; N1 and N2 lengths of nucleotides were compared using ANOVA. $p<0.05$ was considered statistically significant. All statistically significant differences are indicated; ${ }^{*} p<0.05,{ }^{* *} p<0.01,{ }^{* * *} p<0.001[12,17-19]$.

\section{Results}

\section{CDR3 spectratyping of TRBV23-1 and TRBV3-1 in cDNA samples from PBMCs of four healthy volunteers}

CDR3 spectratyping of TRBV23-1 showed a 1-bp or 2-bp (out-of-frame) and 3-bp (in-frame) interval length and peak. On the other hand, the CDR3 spectratyping of TRBV3-1 showed a 3-bp (in-frame) interval length and peak (Fig. 2, Table 2). In the nine cloned sequencing results of the TRBV23-1 CDR3 region PCR products that were randomly collected from $\mathrm{H}-4$, four were in-frame with 3-bp insertions, three were out-of-frame with a 1-bp insertion, and two were out-of-frame with 2-bp insertions (Table 3).

\section{CDR3 repertoire characterization of $T R B V 23-1$ and TRBV3-1 in healthy volunteers by HTS}

The cDNA samples were extracted from PBMCs of four healthy volunteers. A total of 509 reads of the TRBV23-1 CDR3 complete sequences of the four samples, including 142 in-frame and 367 out-of-frame sequences,

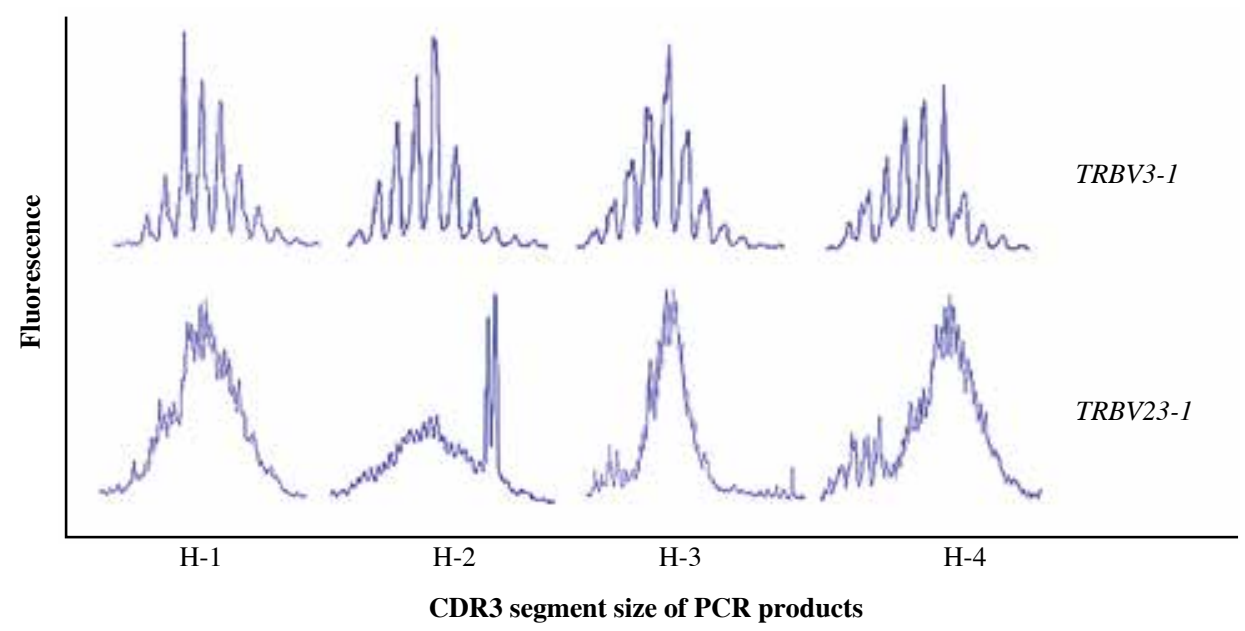

Fig. 2. The CDR3 repertoire (PCR products) spectratyping of TRBV3-1 and TRBV23-1 in the cDNA samples from PBMCs of four healthy volunteers by capillary electrophoresis DNA scanning. Note: TRBV3-1 expressed as regular CDR3 spectratype with 3-bp intervals; TRBV23-1 presented as irregular CDR3 spectratype of small peaks and sloping peaks with 1-bp intervals 
Table 2. The CDR3 repertoire (segment sizes of PCR products) of TRBV23-1 and TRBV3-1 in the cDNA samples from PBMCs of four healthy volunteers

\begin{tabular}{|c|c|c|c|c|c|c|c|}
\hline \multicolumn{4}{|c|}{ TRBV3-1 (bp) } & \multicolumn{4}{|c|}{ TRBV23-1 (bp) } \\
\hline \multirow{2}{*}{$\begin{array}{l}\text { H-1 } \\
185\end{array}$} & \multirow{2}{*}{$\begin{array}{l}\text { H-2 } \\
186\end{array}$} & \multirow{2}{*}{$\begin{array}{c}\text { H-3 } \\
189\end{array}$} & \multirow{2}{*}{$\begin{array}{l}\text { H-4 } \\
189\end{array}$} & \multirow{2}{*}{$\begin{aligned} \text { H-1 } \\
180\end{aligned}$} & \multirow{2}{*}{$\begin{array}{r}\text { H-2 } \\
189190\end{array}$} & \multirow{2}{*}{$\begin{array}{r}\text { H-3 } \\
170171\end{array}$} & H-4 \\
\hline & & & & & & & $171 \quad 173$ \\
\hline 188 & 189 & 192 & 192 & 183184 & 193 & 173 & $174 \quad 176$ \\
\hline 191 & 192 & 195 & 195 & 186187 & 196197 & 176 & $177 \quad 179$ \\
\hline 194195 & 195 & 198 & 198 & 189190191 & 198199200 & 179180 & 180181 \\
\hline 197 & 198 & 201 & 201 & 192 & 202203 & 182183 & 183184 \\
\hline 200 & 201 & 204 & 204 & 195196197 & 206 & 185186187 & \\
\hline 203 & 204 & 207 & 207 & 198199200 & 207208 & 188189190 & 189190191 \\
\hline 206 & 207 & 210 & 210 & 201202203 & & 191192193 & 192193 \\
\hline 209 & 210 & 213 & 213 & 204205206 & & 194195196 & 196197 \\
\hline 212 & 213 & 216 & 216 & 209 & & 197198199 & 198199200 \\
\hline \multirow[t]{5}{*}{215} & 216 & 219 & 219 & 212 & & 200201202 & 201 \\
\hline & 219 & & & & & 203204205 & 204205 \\
\hline & & & & & & $206 \quad 208$ & 207208 \\
\hline & & & & & & 209210 & \\
\hline & & & & & & 212213 & \\
\hline
\end{tabular}

Only the PCR products with $>200$ fluorescent units bands were analyzed by capillary electrophoresis DNA scanning.

The black numbers of TRBV3-1 correspond to CDR3 PCR products of $3 \mathrm{bp}$ interval (according to DNA marker in the experiment), which can show the standard CDR3 spectrum peak pattern having 3 bp interval in Figure 2.

The red numbers of TRBV23-1 correspond to CDR3 PCR products of 3bp interval (according to DNA marker in the experiment). TRBV23-1 black numbers corresponding to CDR3 PCR are larger than the red numbers $1 \mathrm{bp}$. TRBV23-1 blue numbers are larger than the red numbers $2 \mathrm{bp}$, which can show an abnormal CDR3 spectrum peak pattern having 1 bp interval in Figure 2.

were identified. A total of 2,678 reads of the TRBV3-1 CDR3 complete sequences were identified, which included 1,322 in-frame and 1,356 out-of-fame sequences (Table 4). The gDNA samples were extracted from PBMCs of six healthy volunteers. A total of 1,091 CDR3 complete sequence reads were detected in TRBV23-1, which included 350 in-frame reads and 741 out-of-frame reads. A total of 5,043 CDR3 complete sequence reads were detected in TRBV3-1, which included 4,254 in-frame reads and 789 out-of-frame reads (Table 5).

For both cDNA and gDNA, the CDR3 total sequences of $T R B V 23-1$ were significantly smaller than those of the CDR3 sequences of TRBV3-1 (Tables 4 and 5, Fig. 3); the total number of CDR3 sequences of TRBV23-1 out-offrame was significantly higher than that of the TRBV23-1 in-frame sequences (Tables 4 and 5, Fig. 4).

\section{Comparative analysis of the CDR3 repertoire with TRBD and TRBJ usage of TRBV23-1 and TRBV3-1 by HTS}

For both cDNA and gDNA, the usage of TRBD1 in the CDR3 repertoire of $T R B V 3-1$ in-frame sequences was higher than that of TRBD2 in terms of mean percentage; however, it was not statistically significant $(p>0.05)$ (Fig. 5). The usage of TRBD1 in the CDR3 repertoire of $T R B V 23-1$ in-frame and out-of frame sequences was higher than that of TRBD2 and was statistically significant $(p<0.001)$ (Fig. 5). The usage of TRBJ2 was significantly higher than that of $T R B J 1$ in terms of the CDR3 repertoire of all TRBV23-1 and TRBV3-1 $(p<0.001)$ (Fig. 6).

The usage of $T R B D 1$ in the CDR3 repertoire of $T R B V 3-1$ out-of-frame sequences was higher than that of TRBD2 and was statistically significant $(p<0.01)$ in cDNA samples (Fig. 5A). The usage of TRBD1 in the CDR3 repertoire of TRBV3-1 out-of-frame sequences was lower than that of TRBD2 and was statistically significant $(p<0.001)$ for the gDNA samples (Fig. 5B).

\section{Comparative analysis of the $\mathrm{CDR} 3$ repertoire lengths with $\mathrm{N} 1$ an $\mathrm{N} 2$ of TRBV23-1 and TRBV3-1 by HTS}

For the cDNA samples, the CDR3 repertoire of $T R B V 3-1$ in-frame sequences showed that $\mathrm{N} 2$ was longer than N1 in terms of mean length; however, this was not 
Table 3. The TRBV23-1 in-frame and out-of-frame CDR3 gene and amino acid sequences in the cDNA samples from PBMCs of volunteer $\mathrm{H}-4$

\begin{tabular}{|c|c|c|c|c|c|}
\hline \multicolumn{3}{|c|}{ Number TRBV23-1 } & \multirow{2}{*}{$\begin{array}{c}\text { TCR V } \beta \text { CDR3 junctional sequence (BV-N1-BD-N2-BJ) } \\
\text { Q T N Y S N Q P Q H (in-frame) } \\
\text { CAG ACA AAT TAT AGC AAT CAG CCC CAG CAT (in-frame) }\end{array}$} & \multirow{2}{*}{$\begin{array}{c}\text { TRBJ } \\
\text { FG }\end{array}$} & \multirow[b]{2}{*}{ DGTR } \\
\hline $1 / 9$ & ALYL & CASS & & & \\
\hline $1 / 9$ & ALYL & CASR & $\begin{array}{c}\text { H D S N Y G Y T (in-frame) } \\
\text { CAC GAC AGT AAC TAT GGC TAC ACC (in-frame) }\end{array}$ & FG & SGTR \\
\hline $1 / 9$ & ALYL & CASS & $\begin{array}{c}\text { Q V G S G N T I Y (in-frame) } \\
\text { CAG GTG GGA AGT GGA AAC ACC ATA TAT (in-frame) }\end{array}$ & FG & EGSW \\
\hline $1 / 9$ & ALYL & CASS & $\begin{array}{c}\text { Q S P W D L A N Y G Y T (in-frame) } \\
\text { CAA AGC CCC TGG GAT TTA GCT AAC TAT GGC TAC ACC (in-frame) }\end{array}$ & FG & SGTR \\
\hline $1 / 9$ & TVSP & VASS & $\begin{array}{l}\text { Q } * \text { W G K R A V (out-of-frame) } \\
\text { CAA TAG TGG GGA AAG CGA GCA GTA C (out-of-frame) }\end{array}$ & FG & PGTR \\
\hline $1 / 9$ & ALYL & CASR & $\begin{array}{c}\text { Q S G V V E T S S X (out-of-frame) } \\
\text { CAA TCG GGG GTC GTA GAA ACG AGC AGT AG (out-of-frame) }\end{array}$ & FG & PGTR \\
\hline $1 / 9$ & ALYL & CASS & $\begin{array}{c}\text { Q S T S G P * T P G S C X (out-of-frame) } \\
\text { CAA TCG ACT AGC GGC CCC TAA ACA CCG GGG AGC TGT TT (out-of-frame) }\end{array}$ & FG & EGSR \\
\hline $1 / 9$ & ALYL & CASS & $\begin{array}{l}\text { Q S Y H L G Q G E G R D P V X (out-of-frame) } \\
\text { CAATCGTATCACCTGGGACAGGGAGAGGGAAGAGACCCAGTAC (out-of-frame) }\end{array}$ & FG & PGTR \\
\hline $1 / 9$ & ALYL & CASS & $\begin{array}{l}\text { Q D * R E D R G A X (out-of-frame) } \\
\text { CAA GAC TAG CGG GAG GAC CGG GGA GCT T (out-of-frame) }\end{array}$ & FG & EGSR \\
\hline
\end{tabular}

Table 4. The CDR3 repertoire total gene sequence and proportion of TRBV3-1 and TRBV23-1 in-frame and out-of-frame in the cDNA samples from PBMCs of four healthy volunteers by 454 GS FLX HTS

\begin{tabular}{ccccccc}
\hline & Samples name & \multicolumn{2}{c}{ TRBV3-1 CDR3 repertoire } & \multicolumn{2}{c}{ TRBV23-1 CDR3 repertoire } \\
\hline & Total sequences & In-frame (\%) & Out-of-frame (\%) & Total sequences & In-frame (\%) & Out-of-frame (\%) \\
\hline H-1 & 447 & $222(49.7)$ & $225(53.3)$ & 107 & $33(30.8)$ & $74(69.2)$ \\
\hline H-2 & 787 & $379(48.2)$ & $408(51.8)$ & 152 & $31(20.4)$ & $121(79.6)$ \\
\hline H-3 & 493 & $261(52.9)$ & $232(47.1)$ & 89 & $29(32.6)$ & $60(67.4)$ \\
\hline H-4 & 951 & $460(48.4)$ & $491(51.6)$ & 161 & $49(30.4)$ & $112(69.6)$ \\
\hline Total & 2678 & $1322(49.4)$ & $1356(50.6)$ & 509 & $142(27.9)$ & $367(72.1)$ \\
\hline
\end{tabular}

Table 5. The CDR3 repertoire total gene sequence and proportion of TRBV3-1 and TRBV23-1 in-frame and out-of-frame in the gDNA samples from PBMCs of six healthy volunteers by Illumina HTS

\begin{tabular}{ccccccc}
\hline & Sample name & \multicolumn{2}{c}{ TRBV3-1 CDR3 repertoire } & \multicolumn{2}{c}{ TRBV23-1 CDR3 repertoire } \\
\hline & Total sequences & In-frame (\%) & Out-of-frame (\%) & Total sequences & In-frame (\%) & Out-of-frame (\%) \\
\hline H-5 & 757 & $625(82.6)$ & $132(17.4)$ & 161 & $48(29.8)$ & $113(70.2)$ \\
\hline H-6 & 747 & $615(82.3)$ & $132(17.7)$ & 150 & $44(29.3)$ & $106(70.7)$ \\
\hline H-7 & 1318 & $1157(87.8)$ & $161(12.2)$ & 259 & $95(36.7)$ & $164(63.3)$ \\
\hline H-8 & 968 & $856(88.4)$ & $112(11.6)$ & 200 & $66(33)$ & $134(67)$ \\
\hline H-9 & 428 & $341(79.7)$ & $87(20.3)$ & 111 & $36(32.4)$ & $75(67.6)$ \\
\hline H-10 & 825 & $660(80.0)$ & $165(20.0)$ & 210 & $61(29.0)$ & $149(71.0)$ \\
\hline Total & 5043 & $4254(84.4)$ & $789(15.6)$ & 1091 & $350(32.1)$ & $741(67.9)$ \\
\hline
\end{tabular}


A

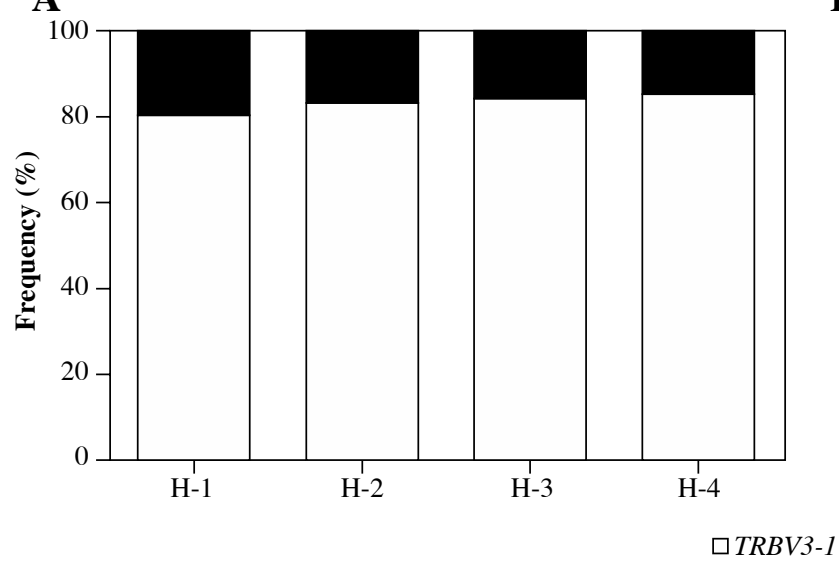

B

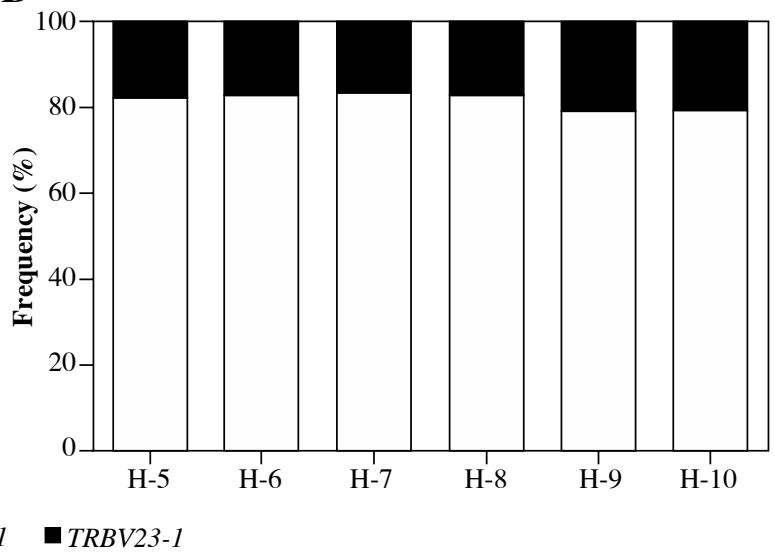

Fig. 3. The CDR3 repertoire sequences proportion of TRBV3-1 and TRBV23-1 by HTS. A) The cDNA samples from PBMCs of four volunteers. B) The gDNA samples from PBMCs of six volunteers

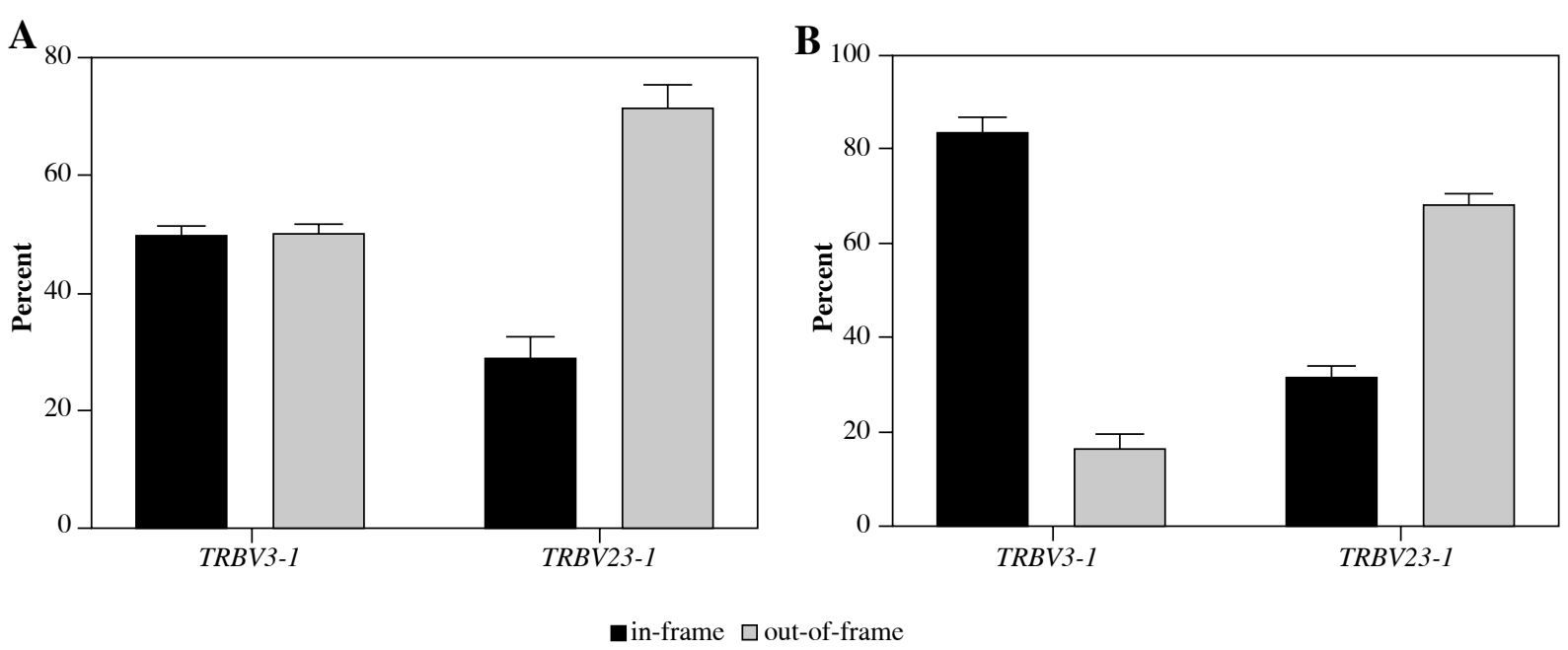

Fig. 4. The CDR3 repertoire sequences proportion of TRBV3-1 and TRBV23-1 in-frame and out-of-frame by HTS. A) The cDNA samples from PBMCs of four volunteers. B) The gDNA samples from PBMCs of six volunteers

statistically significant $(p>0.05)$. The CDR3 repertoire of $T R B V 23-1$ in-frame sequences showed that $\mathrm{N} 2$ was longer than $\mathrm{N} 1$ and was statistically significant $(p<0.05)$. The CDR3 repertoire of TRBV3-1 and TRBV23-1 out-of-frame sequences consistently showed that $\mathrm{N} 2$ was longer than N1 and was statistically significant $(p<0.001)$ (Fig. 7A). However, the CDR3 repertoire of all TRBV23-1 and TRBV3-1 sequences showed no differences in the lengths of $\mathrm{N} 1$ and $\mathrm{N} 2$ for the gDNA samples $(p>0.05)$ (Fig. 7B).

\section{Discussion}

Several studies have shown that human TRBV19S1 (TRBV23-1) is a pseudogene [5, 7]; however, it is also possible that this gene may have undergone rearrangements as a functional TCR [6]. The IMGT and EMBL initially de- fined the T-cell receptor beta chain variable region (clone HVB10.1) as a pseudogene, which was later described as an $O R F$ because the nucleotide bases $G T$ were replaced by $A T$ in the donor splice site, whereas only a portion of the V-GENE has been identified $[3,4]$. The characteristics of CDR3 repertoire rearrangement by $T R B V 23-1$ and the differences in the function of $T R B V$ genes require further analysis.

We compared the CDR3 repertoire rearranged by TRBV23-1 to those rearranged by a random selecting functional gene (TRBV3-1) in cDNA and gDNA samples from PBMCs of healthy volunteers. We determined that: (1) the CDR3 spectratyping of TRBV23-1 (cDNA samples from four volunteers) showed with 1-bp or 2-bp (out-offrame) and 3-bp (in-frame) interval lengths and peaks (Fig. 2, Table 2). However, the CDR3 spectratyping of TRBV3-1 showed with 3-bp (in-frame) interval length and 

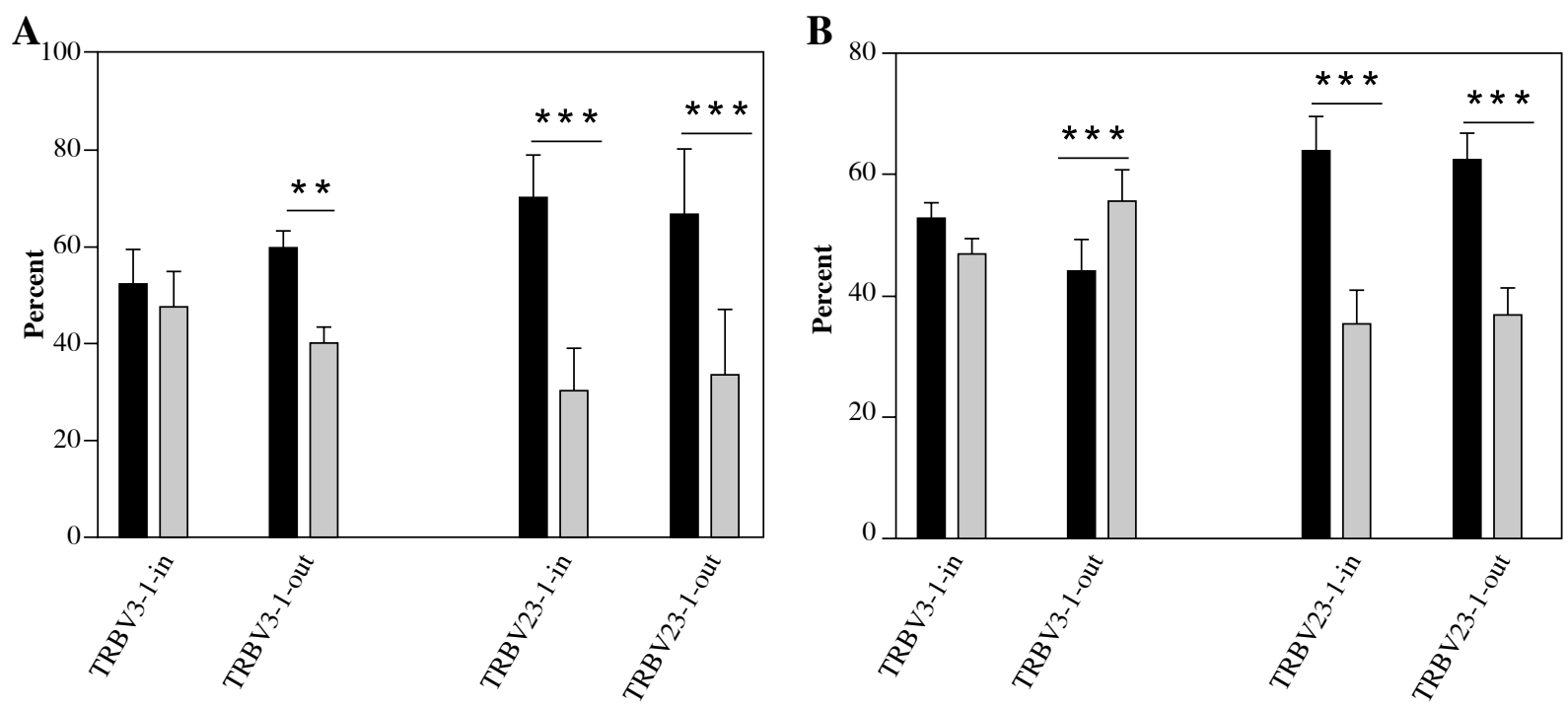

-TRBD1 $\square$ TRBD2

Fig. 5. The CDR3 repertoire with TRBD1 and TRBD2 usage of TRBV3-1 and TRBV23-1 in-frame and out-of-frame by HTS A) The cDNA samples from PBMCs of four volunteers. B) The gDNA samples from PBMCs of six volunteers. Note: The $p$-values were determined using the $\chi^{2}$ test. All statistically significant differences are indicated. ${ }^{* *} p<0.01$, ${ }^{* * * *} p<0.001$
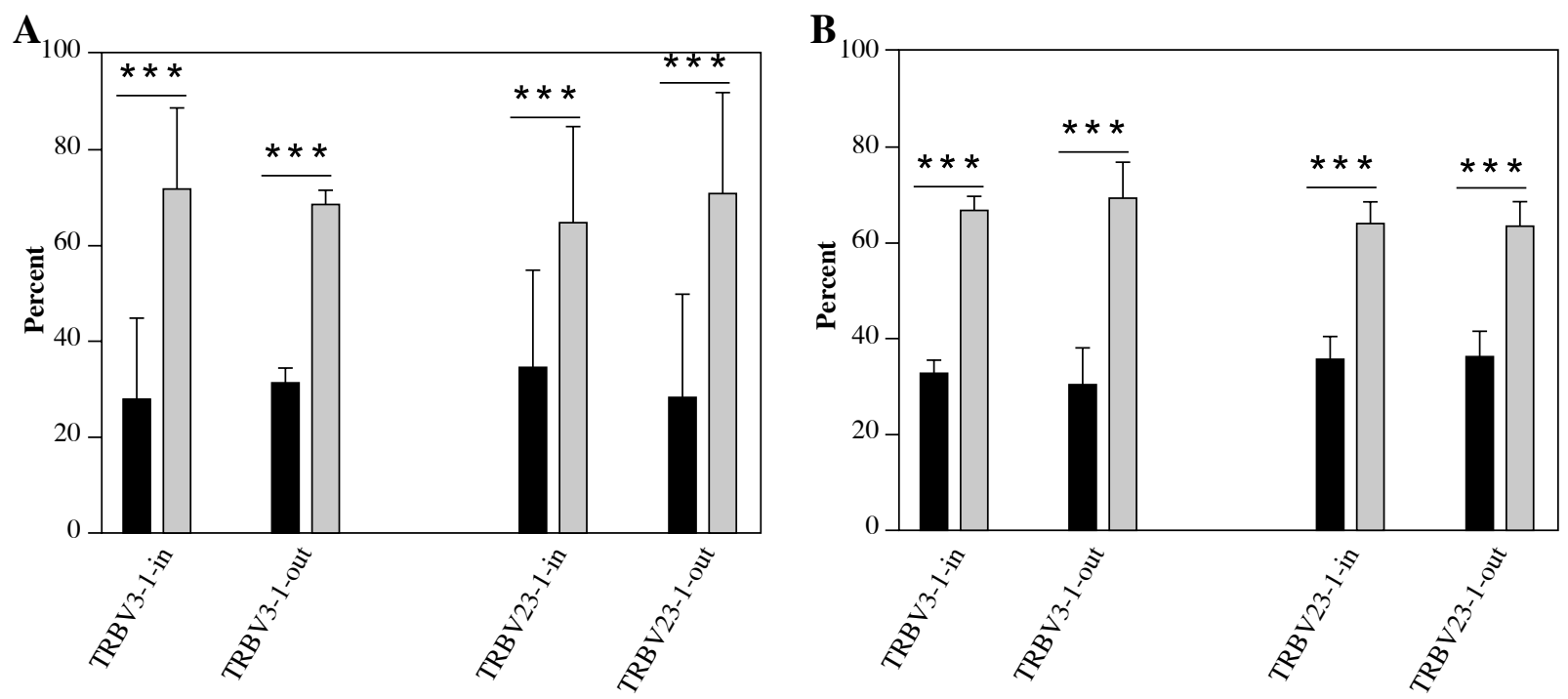

- $T R B J 1 \quad \square T R B J 2$

Fig. 6. The CDR3 repertoire with TRBJ usage of TRBV3-1 and TRBV23-1 in frame and out frame by HTS. A) The cDNA samples from PBMCs of four volunteers. B) The gDNA samples from PBMCs of six volunteers. Note: The $p$-values were determined using the $\chi^{2}$ test. All statistically significant differences are indicated. ${ }^{* * *} p<0.001$

peak (Fig. 2, Table 2); (2) the TRBV23-1 CDR3 region PCR products were randomly selected from H-4 (cDNA samples), four sequences in the CDR3 region were inframe with 3-bp insertions, three sequences in the CDR3 region were out-of-frame with a 1-bp insertion, and two were out-of-frame with 2-bp insertions (Table 3); (3) the CDR3 repertoire of TRBV23-1 was expressed at significantly lower levels than that of TRBV3-1 (Tables 4 and 5); 
A

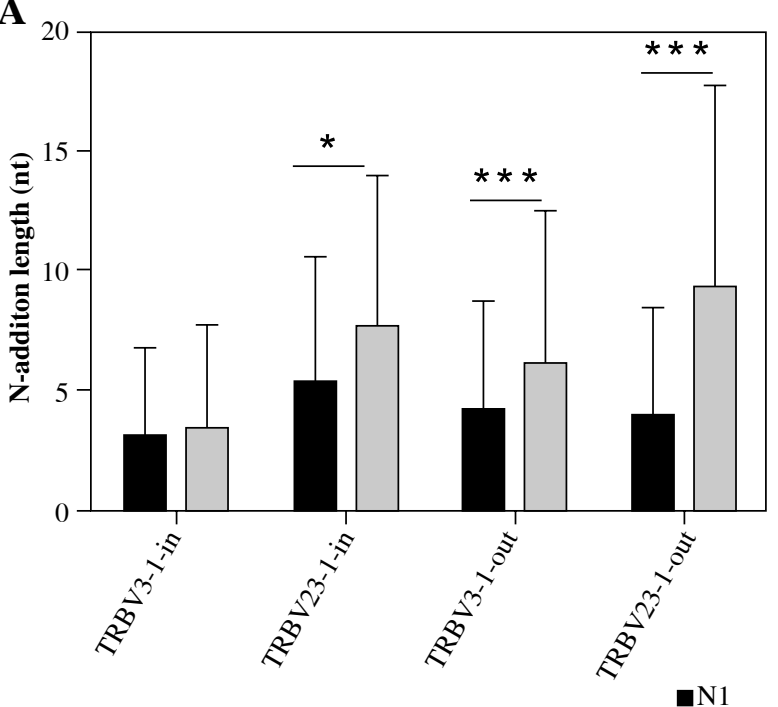

A

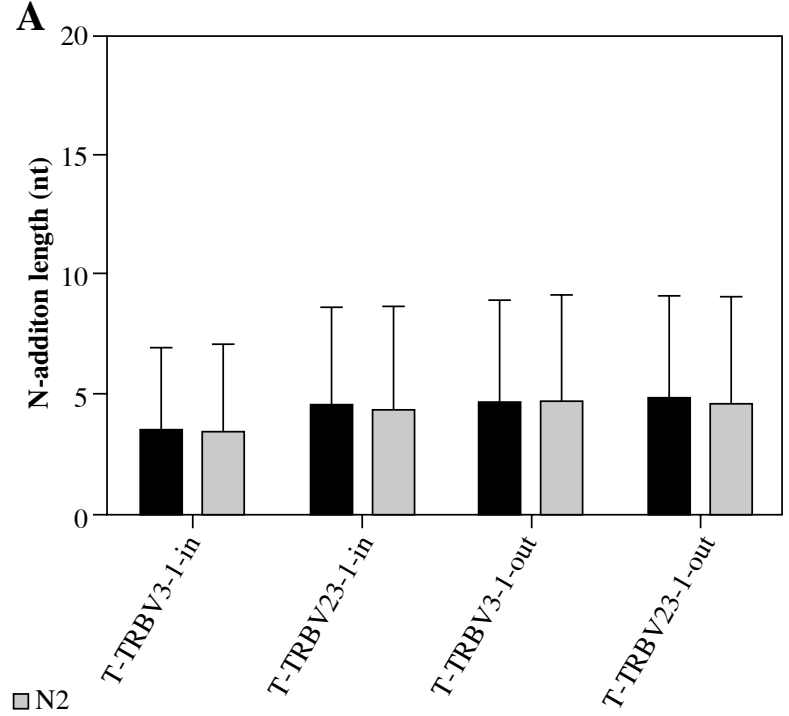

Fig. 7. The CDR 3 repertoire $\mathrm{N} 1$ and $\mathrm{N} 2$ lengths of TRBV3-1 and TRBV23-1 in-frame and out-of-frame by HTS. A) The cDNA samples from PBMC of four volunteers. B) The gDNA samples from PBMC of six volunteers. Note: The $p$-values were determined by using ANOVA. All statistically significant differences are indicated. ${ }^{*} p<0.05,{ }^{* * *} p<0.001$

(4) the CDR3 repertoire of TRBV23-1 consisted of 2/3 outof-frame sequences and $1 / 3$ in-frame sequences, which was exactly the opposite to that observed of the TRBV3-1 inframe and out-of-frame CDR3 repertoire. These findings suggest that the CDR3 repertoire that was rearranged by TRBV23-1 mainly emerged from random rearrangements (2/3 sequences were 1-bp and 2-bp insertions, whereas $1 / 3$ of the sequences harbored 3-bp insertions), and the CDR3 repertoire rearranged by $T R B V 23-1$ may have rendered non-functionality, as well as in the absence of positive and negative selection. The CDR3 repertoire rearranged by $T R B V 3-1$ was mainly composed of an in-frame CDR3, and a few out-of-frame CDR3 regions. In the gDNA samples, the percent of in-frame CDR 3 regions was $>2 / 3$, and the percentage of in-frame CDR3 regions was $<2 / 3$, which may be attributable to cDNA samples that were not amplified by multiplex PCR. In our other experiments on functional TCR repertoires, the percentage of in-frame CDR3 in both human and mice was $>2 / 3$ (cDNA samples and gDNA samples). The results from these massive CDR3 repertoire sequences from TRBV23-1 and TRBV3-1 are in agreement with those of Burkhard's research, which used a small amount of cloning sequencing data to compare and analyze $T R B V 19$ and $T R B V 20$ [7].

The maintenance of $T R B V$ functional gene and $O R F$ (or pseudogene) out-of-frame CDR3 sequences at a certain proportion in peripheral blood might be possibly due to the TCR $\beta$ chain undergoing rearrangements, with the V-D-J combination rearrangement occurring first in one chromosome. Theoretically, the rearrangement of the $T R B V$ functional gene, pseudogene and $O R F$ is random [20]. In the
CDR3 repertoire formed by rearrangements, the CDR3 region insertion/splicing is random, where the ratio of 1-bp, 2 -bp, and 3-bp change is $1 / 3$, respectively. Because $T R B V$ $O R F s$ or pseudogenes do not have functional protein expression after rearrangement, the cells that undergo TRBV $O R F$ or pseudogene rearrangements would either die or initiate a second V-D-J rearrangement in another chromosome. If the second rearrangement were successful, the cells would enter the subsequent selection and maturation stages. If not, then the cells would eventually die. Hence, because TRBV ORFs or pseudogenes are not functionally expressed after rearrangement, these will not be subjected to positive and negative selections to mature. In theory, the total CDR3 repertoire of the TRBV ORF or pseudogene is eventually formed after initiation of rearrangements in another chromosome and successful functional selections. Therefore, the total CDR3 repertoire of the TRBV ORF or pseudogene follows the rearrangement rules of base insertion/deletion, thereby yielding the in-frame ratio of approximately $1 / 3$ (3-bp insertion/deletion) and the outof-frame ratio of approximately 2/3 (1-bp and 2-bp insertion/deletion). At the same time, because TRBV ORF or pseudogenes have no functionality after rearrangement, the formation of their peripheral CDR3 repertoire depends on successful rearrangements involving the $T R B V$ functional gene in another chromosome. Consequently, the number of CDR3 sequences of the TRBV ORF or pseudogene with no functionality should be much lower than that of $T R B V$ functional genes. However, $T R B V$ functional genes would only mature after random rearrangement and positive and negative selections. Theoretically, the ratio of $T R B V$ func- 
tional gene in-frame CDR3 and out-of-frame CDR3 sequences is extremely complex and closely related to individualized selection $[5,21]$. The pattern of rearrangement and selection of TRBV23-1 CDR3 repertoire detected by HTS is consistent with the theory, and these results suggest that we can detect the CDR3 repertoires of the $O R F$ or pseudogene by HTS as a control to analyze the mechanism underlying rearrangements and positive/negative selection of functional genes.

We then compared the usage of TRBD and TRBJ in TRBV23-1 and TRBV3-1 CDR3 repertoires, and the CDR3 repertoire with $T R B D 1$ usage in the TRBV23-1 in-frame and out-of-frame was higher than that of TRBD2, whereas TRBV3-1 in-frame and out-of-frame CDR3 repertoires differed. These findings suggest that the mechanism underlying TRBD rearrangement with TRBV3-1 (F) and TRBV23-1 $(O R F)$ were different, and subsequently yielded variable CDR3 repertoires. However, the usage of TRBJ was similar among these repertoires, and that of TRBJ2 was significantly higher than that of TRBJI in the TRBV23-1 and TRBV3-1 $C D R 3$ repertoire in all the cDNA and gDNA samples from PBMCs of volunteers. Meanwhile, at the TCR loci, the 3' end of TRBV or TRBD in TCR $\beta$ chains is a heptamer (CACAGTG)-23 base pair (bp)-nonamer (ACAAAAACC) rearrangement of signal sequences (3' TRBV 23 RSS and 3' TRBD 23 RSS), whereas the 5' end of TRBD or TRBJ is a nonamer-12bp-heptamer rearrangement of signal sequences (5' TRBD 12 RSS and 5' TRBJ 12 RSS). V-D-J recombination occurs only between segments with the 23 RSS terminal and segments with the 12 RSS terminal, and this restriction is called the $12 / 23$ rule. V-D-J rearrangement of the TCR-beta chain follows the 12/23 rule and the beyond $12 / 23$ restriction. The mechanism of differential gene rearrangement in the TRBV23- 1 and TRBV3-1 CDR3 repertoire may be related to the $12 / 23$ rule and the beyond $12 / 23$ restriction, but that needs further study.

The diversity of the TCR CDR3 repertoire is the result of random combinations, insertions, and splicing of the germline VDJ gene fragment. The random nucleotide insertion ( $\mathrm{N}$ region) was very important for composition of TCR $\beta$ CDR3 diversity during the TCR $\beta$ gene combination process. The region in which the nucleotide fragments randomly added into the V $\beta$-D $\beta$ junction was called the $\mathrm{N} 1$ region. The $\mathrm{N} 2$ region was where the nucleotide fragments randomly added into the $\mathrm{D} \beta$-J $\beta$ junction. We also compared the length of $\mathrm{N} 1$ and $\mathrm{N} 2$ in TRBV23-1 and TRBV3-1 $C D R 3$ repertoires. In the four cDNA samples from PBMCs, the TRBV23-1 in-frame and out-of-frame CDR3 repertoire showed that $\mathrm{N} 2$ was longer than $\mathrm{N} 1$ and similar to the TRBV3-1 out-of-frame CDR3 repertoire, whereas in the gDNA samples from PBMC of six volunteers, the TRBV23-1 and TRBV3-1 in-frame CDR3 and out-of-frame $\mathrm{CDR} 3$ repertoire showed no difference in the lengths of $\mathrm{N} 1$ and $\mathrm{N} 2$. These results show that the insertions during rearrangement of TRBV23-1 and TRBV3-1 (gDNA samples) were similar, whereas in the cDNA samples, the length of $\mathrm{N}$ in unproductive repertoires, namely, TRBV3-1 out-offrame, TRBV23-1 in-frame and TRBV23-1 out-of-frame, showed the same N2 $>\mathrm{N} 1$, while the length of N2 and N1 in the productive repertoire $T R B V 3-1$ was similar, thereby suggesting that the long N2 was closely related to the unproductive TCR receptor.

Taken together, the results of the present study generate the following conclusions: (1) The TRBV23-1 (ORF) CDR3 repertoire expression frequency is lower than that of the TRBV3-1 (F) CDR3 repertoire, indicating that the non-functional rearrangements of TRBV23-1 limit the probabilities of being selected for taking part in rearrangement and development-maturation. (2) Due to the lack of functional maturation, the ratio of TRBV23-1 (ORF) inframe CDR3 and out-of-frame CDR3 completely follows the theoretical rules of random rearrangement. In contrast, the TRBV3-1 (F) in-frame CDR3 and out-of-frame CDR3 ratio, although following the theoretical rules of random rearrangement, must reach maturity through individual negative and positive selection, thereby resulting in complicated and variable ratio characteristics. (3) In the cDNA of the CDR3 repertoire, due to the lack of selection by functional maturation of TRBD gene usage, N-region insertion/splicing of TRBV23-1 (ORF) in-frame and outof-frame CDR3 present characteristics that are similar to the TRBV3-1 (F) out-of-frame CDR3 repertoire (non-functional).

In summary, these results indicate that analysis of the composition and characteristics of the TRBV23-1 (ORF) CDR3 repertoire could provide the basis for research on the rules and mechanisms of TCR random rearrangement and the positive or negative selection process. At the same time, it could provide experimental quality control and research references for the analysis of the $T R B V$ functional gene CDR3 repertoire.

\section{Acknowledgements}

We are grateful to the ten healthy volunteers for supporting this study. We thank Adaptive Biotechnologies Corporation (Seattle, WA, US) and Majorbio Bio-pharm Technology Co., Ltd (Shanghai, China) for help with human TCR $\beta$ chain CDR3 repertoire sequencing and analysis.

The work was supported by grants from the National Prophase Project on Basic Research of China $(973$ pre-Program, 2008CB517310), the Natural Science and International Cooperation Program of Guizhou Province (2008-700105 \& 2007-2122) and the National Natural Science Foundation of China (31160195 and 81441048).

The authors declare no conflict of interest. 


\section{References}

1. Tonegawa S (1988): Antibody and T-cell receptors. JAMA 259: $1845-1847$.

2. Rowen L, Koop BF, Hood L (1996): The complete 685-kilobase DNA sequence of the human beta $\mathrm{T}$ cell receptor locus. Science 272: 1755-1762.

3. http://www.imgt.org/

4. http://www.embl.org/

5. Currier JR, Yassai M, Robinson MA, Gorski J (1996): Molecular defects in TCRBV genes preclude thymic selection and limit the expressed TCR repertoire. J Immunol 157: 170-175.

6. Than S, Kharbanda M, Chitnis V, et al. (1999): Clonal dominance patterns of CD8 T cells in relation to disease progression in HIV-infected children. J Immunol 162: 3680-3686.

7. Manfras BJ, Terjung D, Boehm BO (1999): Non-productive human TCR beta chain genes represent V-D-J diversity before selection upon function: insight into biased usage of TCRBD and TCRBJ genes and diversity of CDR3 region length. Hum Immunol 60: 1090-1100.

8. Pommié C, Levadoux S, Sabatier R, et al. (2004): IMGT standardized criteria for statistical analysis of immunoglobulin V-REGION amino acid properties. J Mol Recognit 17: 17-32.

9. Alamyar E, Duroux P, Lefranc MP, Giudicelli V (2012): IMGT(R) tools for the nucleotide analysis of immunoglobulin (IG) and T cell receptor (TR) V-(D)-J repertoires, polymorphisms, and IG mutations: IMGT/V-QUEST and IMGT/ HighV-QUEST for NGS. Methods Mol Biol 882: 569-604.

10. Alamyar E, Giudicelli V, Li S, et al. (2012): IMGT/HighVQUEST: the IMGT® web portal for immunoglobulin (IG) or antibody and T cell receptor (TR) analysis from NGS high throughput and deep sequencing. Immun Res 8: 26.

11. Li S, Lefranc MP, Miles JJ, et al. (2013): IMGT/HighV QUEST paradigm for T cell receptor IMGT clonotype diversity and next generation repertoire immunoprofiling. Nat Commun 4: 2333.

12. Robins HS, Campregher PV, Srivastava SK, et al. (2009): Comprehensive assessment of T-cell receptor beta-chain diversity in alpha beta T cells. Blood 114: 4099-4107.

13. Robins HS, Srivastava SK, Campregher PV, et al. (2010): Overlap and effective size of the human CD8+ T cell receptor repertoire. Sci Transl Med 2: 47ra64.

14. Sherwood AM, Desmarais C, Livingston RJ, et al. (2011): Deep sequencing of the human TCR $\gamma$ and TCR $\beta$ repertoires suggests that TCR $\beta$ rearranges after $\alpha \beta$ and $\gamma \delta$ T cell commitment. Sci Transl Med 3: 90ra61.

15. Wu D, Sherwood A, Fromm JR, et al. (2012): High-throughput sequencing detects minimal residual disease in acute T lymphoblastic leukemia. Sci Transl Med 4: 134ra63.

16. http://www.immunoseq.com

17. Stewart JJ, Lee CY, Ibrahim S, et al. (1997): A Shannon entropy analysis of immunoglobulin and T cell receptor. Mol Immunol 34: 1067-1082.

18. Yousfi Monod M, Giudicelli V, Chaume D, Lefranc MP (2004): IMGT/JunctionAnalysis: the first tool for the analysis of the immunoglobulin and $\mathrm{T}$ cell receptor complex V-J and V-D-J JUNCTIONs. Bioinformatics 20 Suppl 1: i379-385.

19. Lefranc MP (2011): IMGT unique numbering for the variable $(\mathrm{V})$, constant $(\mathrm{C})$, and groove $(\mathrm{G})$ domains of $\mathrm{IG}, \mathrm{TR}, \mathrm{MH}$, IgSF, and MhSF. Cold Spring Harb Protoc 2011; 633-642.
20. Meyer-Olson D, Brady KW, Blackard JT, et al. (2003): Analysis of the TCR beta variable gene repertoire in chimpanzees: identification of functional homologs to human pseudogenes. J Immunol 170: 4161-4169.

21. Murphy KM (2011): Janeway's immunobiology. Garland Science, New York. 